\title{
Comprehensive gene expression analysis of canine invasive urothelial bladder carcinoma by RNA-Seq
}

Shingo Maeda ${ }^{1 *}{ }^{*}$, Hirotaka Tomiyasu ${ }^{2 \dagger}$, Masaya Tsuboi ${ }^{3}$, Akiko Inoue ${ }^{1}$, Genki Ishihara ${ }^{4}$, Takao Uchikai ${ }^{4}$, James K. Chambers ${ }^{3}$, Kazuyuki Uchida ${ }^{3}$, Tomohiro Yonezawa ${ }^{1}$ and Naoaki Matsuki ${ }^{1}$

\begin{abstract}
Background: Invasive urothelial carcinoma (iUC) is a major cause of death in humans, and approximately 165,000 individuals succumb to this cancer annually worldwide. Comparative oncology using relevant animal models is necessary to improve our understanding of progression, diagnosis, and treatment of iUC. Companion canines are a preferred animal model of iUC due to spontaneous tumor development and similarity to human disease in terms of histopathology, metastatic behavior, and treatment response. However, the comprehensive molecular characterization of canine iUC is not well documented. In this study, we performed transcriptome analysis of tissue samples from canine iUC and normal bladders using an RNA sequencing (RNA-Seq) approach to identify key molecular pathways in canine iUC.
\end{abstract}

Methods: Total RNA was extracted from bladder tissues of 11 dogs with iUC and five healthy dogs, and RNA-Seq was conducted. Ingenuity Pathway Analysis (IPA) was used to assign differentially expressed genes to known upstream regulators and functional networks.

Results: Differential gene expression analysis of the RNA-Seq data revealed 2531 differentially expressed genes, comprising 1007 upregulated and 1524 downregulated genes, in canine iUC. IPA revealed that the most activated upstream regulator was PTGER2 (encoding the prostaglandin $E_{2}$ receptor EP2), which is consistent with the therapeutic efficiency of cyclooxygenase inhibitors in canine iUC. Similar to human iUC, canine iUC exhibited upregulated ERBB2 and downregulated TP53 pathways. Biological functions associated with cancer, cell proliferation, and leukocyte migration were predicted to be activated, while muscle functions were predicted to be inhibited, indicating muscle-invasive tumor property.

Conclusions: Our data confirmed similarities in gene expression patterns between canine and human iUC and identified potential therapeutic targets (PTGER2, ERBB2, CCND1, Vegf, and EGFR), suggesting the value of naturally occurring canine iUC as a relevant animal model for human iUC.

Keywords: Dog, Transitional cell carcinoma, Animal model, RNA sequencing

\footnotetext{
* Correspondence: amaeda@mail.ecc.u-tokyo.ac.jp

${ }^{\dagger}$ Equal contributors

${ }^{1}$ Department of Veterinary Clinical Pathobiology, Graduate School of

Agricultural and Life Sciences, The University of Tokyo, Tokyo, Japan

Full list of author information is available at the end of the article
}

(c) The Author(s). 2018 Open Access This article is distributed under the terms of the Creative Commons Attribution 4.0 International License (http://creativecommons.org/licenses/by/4.0/), which permits unrestricted use, distribution, and reproduction in any medium, provided you give appropriate credit to the original author(s) and the source, provide a link to the Creative Commons license, and indicate if changes were made. The Creative Commons Public Domain Dedication waiver (http://creativecommons.org/publicdomain/zero/1.0/) applies to the data made available in this article, unless otherwise stated. 


\section{Background}

Urothelial carcinoma of the bladder is a common malignancy with 165,000 estimated global human deaths per year [1]. This cancer is heterogeneous and histologically classified into low-grade, superficial type and high-grade, invasive type. Low-grade tumors are more common (approximately $70 \%$ ) and are usually associated with a favorable prognosis. In contrast, high-grade, muscle-invasive urothelial carcinoma (iUC) is less prevalent (approximately 30\%) but has a high risk of death from distant metastasis [2]. Cisplatinbased chemotherapy is the standard first-line treatment of metastatic iUC and provides overall survival benefits [3]. Meanwhile, up to two-thirds of patients cannot tolerate the regimens due to nephrotoxicity [4]. There have been little advances in the treatment of iUC in the past 20 years. Thus, better management of iUC is urgently required.

Our understanding of various cancers has benefited greatly from comparative oncology using relevant animal models, especially mouse models. Several experimentally induced mouse models of bladder cancer have been established, including carcinogen-based models, engraftment models, and genetically engineered models. However, in contrast to many other cancer types, relatively few mouse models of bladder cancer have been described, and in particular, few models display muscleinvasive or metastatic cancer phenotypes [5]. Therefore, more suitable iUC animal models are crucial.

Naturally occurring iUC in pet dogs closely resembles human iUC with regard to clinical signs, histopathology, heterogeneity, metastatic behavior, disease progression, and response to cisplatin-based chemotherapy [6, 7]. Bladder carcinoma makes up approximately $2 \%$ of all spontaneous cancers in dogs, which is similar to its incidence in humans [1, 7]. However, unlike humans, the vast majority of canine bladder carcinomas are highgrade iUC (> 90\% of cases); low-grade, superficial tumors are uncommon in dogs. Metastases of lymph nodes or distant regions (e.g., lungs, liver, kidneys) have been reported in approximately $15 \%$ of dogs at diagnosis and in $40-50 \%$ at death [6]. Moreover, clinical trials of canine iUC can be conducted in a relatively short period because dogs have a shorter life span than humans. Thus, dogs with iUC offer an ideal model to evaluate novel anti-cancer drugs. However, canine iUC has not been well characterized at the molecular level. Only one study, based on microarray analysis, has shown similarities in gene expression patterns between dogs and humans with iUC [8]. Although this previous study revealed the involvement of certain genes (TP53 and EGFR), other pathways related to carcinogenesis were not mentioned. To our knowledge, no detailed gene expression analyses using RNA sequencing (RNA-Seq) in canine iUC have been reported to date. RNA-Seq allows more sensitive detection of transcripts than microarrays in both dogs and humans $[9,10]$, contributing to a better understanding of molecular pathways in iUC.

In the present study, we performed a comprehensive gene expression analysis of canine iUC by RNA-Seq to identify key molecular pathways in canine iUC. The findings from dogs with iUC have the potential to improve our understanding of progression, diagnosis, and treatment of iUC in humans.

\section{Methods}

\section{Ethics statement}

The study protocol of tumor tissue sampling was approved by the animal care committee of the Veterinary Medical Center of the University of Tokyo, and written informed consent was obtained from all dog owners. The use of experimental animals in this study was approved by the animal care committee of the University of Tokyo (approval no. P11-530).

\section{Tissue samples}

Tumor tissue specimens were obtained from 11 dogs with naturally occurring iUC by total cystectomy at the Veterinary Medical Center of the University of Tokyo. No cases received chemotherapy or radiation prior to tissue sampling. Each tumor tissue was macroscopically obtained by a pathologist with Japanese College of Veterinary Pathologists board certification (M.T.) and was fixed in $10 \%$ neutral buffered formalin for $24 \mathrm{~h}$ prior to embedding in paraffin. Histopathological examination confirmed a diagnosis of high-grade iUC for all cases. Each case was categorized by histology according to the World Health Organization criteria [11, 12]. Small pieces of tumor specimens were collected and snapfrozen in liquid nitrogen and stored at $-80{ }^{\circ} \mathrm{C}$ for RNA extraction. Urine samples were collected from all dogs using a urethral catheter, and BRAF V595E mutation was assessed by digital PCR assay [13].

Archived snap-frozen normal bladder tissues obtained from five healthy beagles euthanized for other experimental purposes were used as a control. This group included two females (all intact) and three males (all intact), aged 97-130 months (median, 112 months). These dogs exhibited no clinical signs and were not treated with any drugs. Routine urinalysis and blood examinations, including a complete blood count and measurements of blood urea nitrogen, creatinine, alanine aminotransferase, and alkaline phosphatase levels, showed no abnormalities.

\section{RNA-Seq}

Total RNA was extracted from tumor and normal bladder tissues using the RNeasy Mini Kit (Qiagen, Valencia, CA). RNA integrity was examined with an Agilent 2100 Bioanalyzer (Agilent Technologies, Santa 
Clara, CA) and RNA integrity number (RIN) values of all samples were $>7$. Sequencing libraries were prepared with the TruSeq Stranded mRNA Library Prep Kit for NeoPrep (Illumina, San Diego, CA). RNA-Seq (75-bp paired-end) using NextSeq 500 (Illumina) with the High Output Kit (Illumina) was conducted, and a minimum of 35 million read-pairs was generated for each sample.

Initial quality control of RNA-Seq data (FASTQ) for each sample was performed using the FastQC software (version 0.11.5; http://www.bioinformatics.babraham.ac. $\mathrm{uk} /$ projects/fastqc). FASTQ data were trimmed with Trimmomatic (version 0.36) [14]. Bowtie2 (version 2.2.9) [15] and TopHat (version 2.0.14) were used to map paired-end reads to canine genomes (CanFam3.1) and analyze mapping results to identify splice junctions between exons. Transcript abundance was estimated using Cufflinks [16] with gene transfer file (CanFam3.1.86). Fragments per kilobase of transcript per million mapped reads (FPKM) was imported into $\mathrm{R}$ (version 3.3.2), and principal component analysis (PCA) was conducted with genes for which the sum of FPKMs of all samples was > 10. Next, gene counts for each sample were imported into $\mathrm{R}$ for differential gene expression analysis with EdgeR [17, 18]. TMM normalization [19] and Tagwise dispersion (individual dispersion for each gene) were used to adjust for abundance differences across samples, and differentially expressed genes (DEGs) were extracted. The normalized gene counts were imported into Cluster3.0 and Java TreeView (version 1.1.6r4) for hierarchical clustering analysis and visualization. Enriched pathway and functional classification analyses of differences in expression of DEGs were performed using Ingenuity ${ }^{\oplus}$ Pathway Analysis (IPA ${ }^{\oplus}$, Qiagen). The datasets used and/or analysed during the current study are available from the corresponding author on reasonable request and will also be available at the DDBJ Sequenced Read Archive repository (http://trace.ddbj.nig.ac.jp/dra/ index_e.html) with accession number DRA005844.

\section{Quantitative real-time PCR}

The mRNA expression levels of 5 DEGs extracted in this study were quantified by 2-step real-time RT-PCR (Thermal Cycler Dice Real Time System; Takara Bio, Shiga, Japan), as described previously [20, 21]. TATA-box binding protein (TBP) was used as a reference gene; primer pair sequences are shown in Additional file 1: Table S1.

\section{Immunohistochemistry}

Immunohistochemistry (IHC) for EP2 was conducted on 4- $\mu$ m-thick paraffin-embedded sections of the 15 iUC tissues including the 11 cases used for RNA-Seq analysis. A rabbit polyclonal antibody against EP2 (1:100 in dilution, Item No. 101750, Cayman Chemical, Ann Arbor,
MI) was used and IHC was performed following previous studies $[22,23]$.

\section{Statistics}

Statistical analyses were performed using JMP 9 (SAS Institute, Cary, NC). Fisher's exact test was used to compare the sex distribution among dogs with iUC and healthy controls. The Mann-Whitney $U$ test was used to compare age and mRNA expression of DEGs between dogs with iUC and healthy controls. Statistical significance was defined as $P<0.05$.

\section{Results}

Clinical and pathological findings in dogs with iUC

All 11 cases had a histopathological diagnosis of highgrade iUC, and characteristics of each case are shown in Table 1 . The dogs comprised 10 females (five intact and five neutered) and one intact male, aged 85-161 months (median, 145 months), with several breeds represented. There were no significant differences in sex and age between the iUC cases and healthy controls. According to the WHO TNM classification for canine urinary bladder cancer [24, 25], tumors in 8/11 (72.7\%) dogs were classified as T2 tumors (muscle invasive) and those in $3 / 11$ (27.3\%) as T3 tumors (tumor invading neighboring organs). Iliac lymph node involvement was detected in 2/ $11(18.2 \%)$ dogs. No distant metastasis (to the lung) was observed in any of the cases at diagnosis, while papillary tumors were observed in 8/11 (72.7\%) dogs. Tumor grade was determined according to most anaplastic nuclear appearance [12]: tumors in 2/11 (18.2\%) dogs were classified as grade 1 (well-differentiated cells), 6/11 (54. $5 \%$ ) as grade 2 (moderately differentiated cells), and 3/11 $(27.3 \%)$ as grade 3 (anaplastic cells). Various types and degrees of inflammation were observed in each tumor microenvironment (Table 1). BRAF V595E mutation was detected in 6/11 (54.5\%) cases.

\section{Principal component analysis}

The differences in gene expression profiles between normal bladder and canine iUC samples are illustrated in the PCA plot (Fig. 1), which clearly separates normal bladder from tumor samples. A previous study revealed that the BRAF V595E mutation is observed at significantly high rates (approximately 80\%) in canine iUC [13]. However, the pathological significance of this mutation remains unclear. Thus, we used PCA to assess the clustering of canine iUC cases with or without BRAF V595E mutation. The PCA plot failed to show a clear separation of cases with or without BRAF mutation (Additional file 2: Figure S1). 
Table 1 Clinical and histological characteristics of dogs with iUC and healthy control dogs

\begin{tabular}{|c|c|c|c|c|c|c|c|c|}
\hline Case No. & Sex & Age (months) & Breed & $\begin{array}{l}\text { TNM stage at } \\
\text { diagnosis }\end{array}$ & $\begin{array}{l}\text { Papillary } \\
\text { histology }\end{array}$ & $\begin{array}{l}\text { Grade } \\
\text { (nuclear atypia) }\end{array}$ & $\begin{array}{l}\text { Type (degree) } \\
\text { of inflammation }\end{array}$ & BRAF mutation \\
\hline iUC1 & $\mathrm{F}$ & 146 & Wire Fox Terrier & T2NOMO & No & Grade 2 & Lyn, Neu (mild) & - \\
\hline iUC2 & FS & 122 & Papillon & T2NOMO & Yes & Grade 2 & Lym (moderate) & + \\
\hline iUC3 & $\mathrm{F}$ & 85 & Miniature Dachshund & T3NOMO & Yes & Grade 2 & Lym (moderate) & + \\
\hline iUC4 & $\mathrm{F}$ & 147 & Papillon & T2NOMO & Yes & Grade 3 & Lym (mild) & - \\
\hline iUC5 & FS & 131 & Miniature Dachshund & T3NOMO & Yes & Grade 3 & Lym, Eo (moderate) & + \\
\hline iUC6 & $\mathrm{F}$ & 122 & Border Collie & T2NOMO & No & Grade 2 & Lym (mild) & - \\
\hline iUC7 & FS & 149 & Miniature Dachshund & T2N1M0 & No & Grade 3 & Lyn, Neu (mild) & + \\
\hline iUC8 & $\mathrm{F}$ & 105 & French Bulldog & T2NOMO & Yes & Grade 3 & Lym (moderate) & + \\
\hline iUC9 & M & 161 & Pembroke Welsh Corgi & T3N1M0 & Yes & Grade 2 & Lym (mild) & - \\
\hline iUC10 & FS & 158 & Toy Poodle & T2NOMO & Yes & Grade 2 & Lym (severe) & + \\
\hline iUC11 & FS & 145 & Mongrel & T2NOMO & Yes & Grade 1 & Lym (mild) & - \\
\hline $\mathrm{HCl}$ & $\mathrm{F}$ & 130 & Beagle & - & - & - & - & - \\
\hline $\mathrm{HC2}$ & $\mathrm{F}$ & 112 & Beagle & - & - & - & - & - \\
\hline $\mathrm{HC} 3$ & M & 97 & Beagle & - & - & - & - & - \\
\hline $\mathrm{HC} 4$ & M & 122 & Beagle & - & - & - & - & - \\
\hline $\mathrm{HC} 5$ & M & 108 & Beagle & - & - & - & - & - \\
\hline
\end{tabular}

iUC invasive urothelial carcinoma, $H C$ healthy control, $F$ female, FS female spayed, $M$ male, Lym lymphocyte, Neu neutrophil, Eo eosinophil

\section{Differential expression analysis}

Differential expression analysis revealed several genes that were differentially regulated in iUC cases when compared with normal controls with a q-value $<0.01$. In total, 2531 DEGs showed significant changes between normal bladder and iUC samples. Of these, 1007 DEGs were upregulated and 1524 were downregulated in iUC dogs with a foldchange $\geq 2$. The 500 most strongly up- and downregulated DEGs are shown in Additional file 3: Table S2 and Additional file 4: Table S3, respectively. Hierarchical clustering of these DEGs showed a similar gene expression pattern among the 11 canine iUC tissues and a clear separation of normal and tumor tissues (Fig. 2). When hierarchical clustering was conducted using the genes that were shown to divide the human iUC patients into subgroups [26], no clear separation was observed between normal controls and canine iUCs (data not shown). Additional hierarchical clustering was conducted using 388 genes that were shown to divide the dogs with iUC into subgroups [8], and three clusters were identified: those composed of five normal controls, two iUCs (TCC1 and TCC11), or remaining nine iUCs (Additional file 5: Figure S2).

To validate our findings from the RNA-Seq analysis, we examined mRNA expression levels of Top 5 DEGs

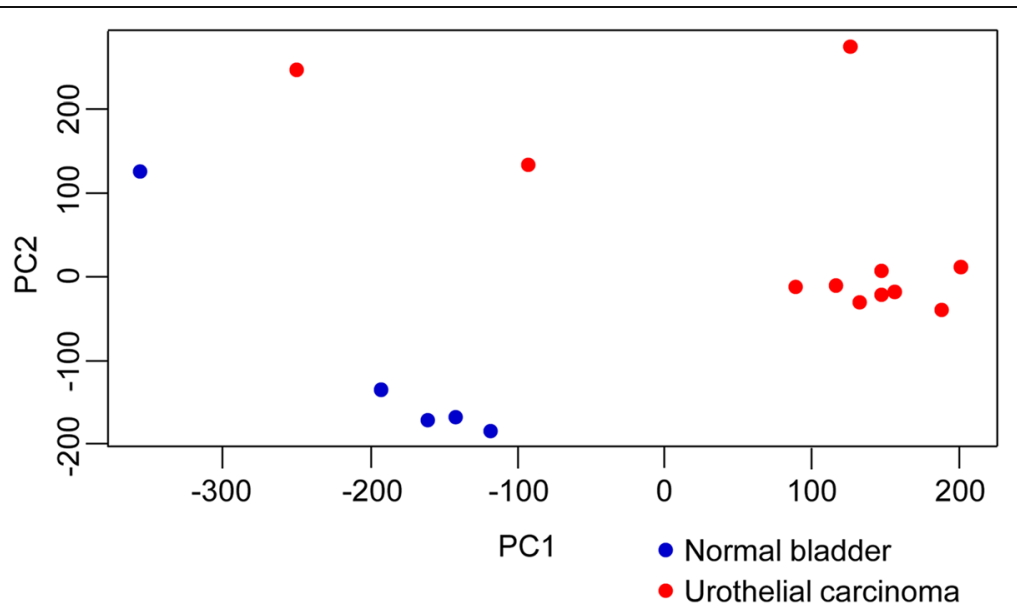

Fig. 1 Principal component analysis (PCA) plot of canine iUC and normal bladder. The PCA plot shows clear separation between canine iUC (red) and normal bladder (blue) samples 


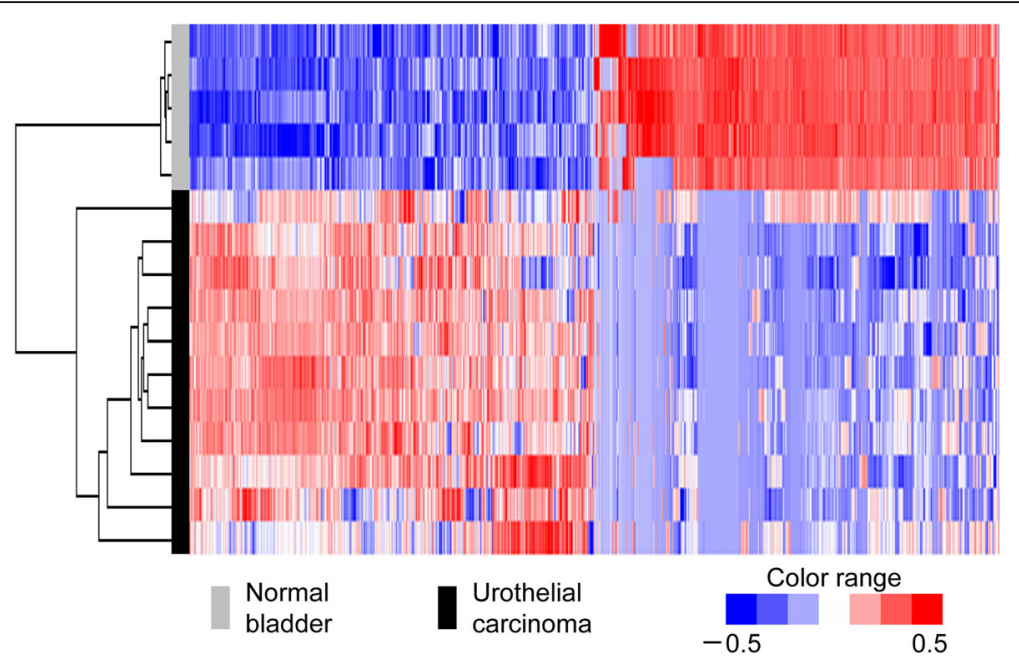

Fig. 2 Hierarchical clustering of differentially expressed genes between canine iUC and normal bladder. Genes indicated in blue are downregulated, while genes indicated in red are upregulated

(CHST4, PIGR, S100A14, ADGRF1, and AGR2) by quantitative PCR and confirmed the trend observed in the RNA-Seq data, whereby there was a significant increase in dogs with iUC compared to that in healthy controls (Additional file 6: Figure S3).

\section{Pathway analysis}

Enriched pathway and functional classification analyses of DEGs were performed using IPA. For simplicity, IPA analysis was conducted with the 500 most strongly up- and downregulated DEGs. Extracted upstream regulators associated with these DEGs are shown in Table 2 and Additional file 7: Table S4. Relative to those in normal bladder, the most significant upstream regulators predicted to be activated in canine iUC were PTGER2 and ERBB2, whereas those predicted to be inhibited were Irgm1, TP53, let-7, and ZFP36 (Table 2). The transcription factors CCND1, FOXM1, E2F3, FOXO1, and JUN as well as proinflammatory and angiogenesis cytokines IL6, IL1B, CSF2, TNF, IFNG, IL22, HGF, and Vegf were among the top 30 upstream regulators predicted to be activated.

We further performed IPA to examine which biological processes are associated with canine iUC. IPA revealed that gene expression in canine iUC tissues was associated with biological processes involving cancer, neoplasia of epithelial tissue, tumorigenesis of tissue, proliferation of cells and cell movement of leukocytes (Table 3). Mammary tumor gene expression was also predicted to be activated, while gene expression related to muscle function and contractility was predicted to be inhibited.

\section{Immunohistochemistry for EP2}

Our RNA-Seq analysis revealed that PTGER2 was the most activated upstream regulator in canine iUC $(P<1$. 15E-17, Table 2). To further examine protein expression,
IHC for EP2, which is encoded by PTGER2, was performed on tumor sections from 15 dogs with histopathologically confirmed iUC. EP2 expression was observed in primary tumor cells and peritumoral inflammatory cells (Fig. 3); EP2 expression was detected in tumor and inflammatory cells in 11/15 (73.3\%) and 2/15 (13.3\%) of dogs, respectively. In contrast, normal epithelial cells did not express EP2 (data not shown).

\section{Discussion}

In this study, we applied a transcriptome sequencing approach to identify gene expression characteristics of canine iUC. We detected 2531 DEGs, and hierarchical clustering of these DEGs showed a clear separation of normal and tumor tissues. Using genes that were previously shown to divide the canine iUC into subgroups [8], the present study also identified two distinct subgroups within dogs with iUC. Furthermore, pathway analysis revealed a number of upstream regulators of the DEGs in canine iUC, providing insights into disease biology and potential targets for therapeutic intervention.

PTGER2, encoding prostaglandin $\mathrm{E}_{2}\left(\mathrm{PGE}_{2}\right)$ receptor 2 (also known as EP2), was the most activated upstream regulator in canine iUC. In addition, we confirmed that EP2 protein was strongly expressed in tumor cells and surrounding inflammatory cells but not in normal epithelial cells. $\mathrm{PGE}_{2}$ is a major arachidonic acid-derived metabolite that is abundantly produced by cyclooxygenase- 2 (COX-2) upon inflammation [27]. The biological effects of $\mathrm{PGE}_{2}$ are mediated through four types of the receptor, EP1-4 [28]. Upregulation of COX-2 accompanied by high levels of $\mathrm{PGE}_{2}$ is common in a variety of tumor tissues [29]. Previous studies have shown that elevated expression of COX-2 and increased levels of $\mathrm{PGE}_{2}$ are observed in both human and canine bladder cancer [30-35]. $\mathrm{PGE}_{2}-$ 
Table 2 Top 30 upstream regulators of differentially expressed genes in canine iUC

\begin{tabular}{|c|c|c|c|c|}
\hline Upstream regulator & Molecule type & Predicted activation state & Bias-corrected z-score & $P$-value of overlap \\
\hline PTGER2 & G-protein coupled receptor & Activated & 4.735 & 1.15E-17 \\
\hline ERBB2 & Kinase & Activated & 4.679 & $9.58 \mathrm{E}-17$ \\
\hline $\operatorname{lrgm} 1$ & Other & Inhibited & -4.175 & $9.40 \mathrm{E}-16$ \\
\hline IL6 & Cytokine & Activated & 3.46 & 3.32E-15 \\
\hline IL1B & Cytokine & Activated & 4.071 & $3.01 \mathrm{E}-13$ \\
\hline CSF2 & Cytokine & Activated & 5.706 & $8.53 \mathrm{E}-13$ \\
\hline CCND1 & Transcription regulator & Activated & 4.073 & $2.25 \mathrm{E}-12$ \\
\hline TP53 & Transcription regulator & Inhibited & -2.977 & $1.41 \mathrm{E}-11$ \\
\hline TNF & Cytokine & Activated & 4.168 & $1.57 \mathrm{E}-11$ \\
\hline FOXM1 & Transcription regulator & Activated & 4.079 & 3.90E-09 \\
\hline HGF & Growth factor & Activated & 3.956 & 6.87E-09 \\
\hline E2F3 & Transcription regulator & Activated & 3.186 & 7.04E-09 \\
\hline FOXO1 & Transcription regulator & Activated & 3.643 & $1.21 \mathrm{E}-08$ \\
\hline NFkB (complex) & Complex & Activated & 3.65 & 2.39E-08 \\
\hline IFNG & Cytokine & Activated & 3.536 & $2.72 \mathrm{E}-08$ \\
\hline let-7 & MicroRNA & Inhibited & -4.119 & $3.16 \mathrm{E}-08$ \\
\hline RABL6 & Other & Activated & 3.197 & $3.28 \mathrm{E}-08$ \\
\hline JUN & Transcription regulator & Activated & 4.005 & 4.11E-08 \\
\hline IL $1 \mathrm{~A}$ & Cytokine & Activated & 3.265 & $1.94 \mathrm{E}-07$ \\
\hline E2f & Group & Activated & 3.226 & $2.36 \mathrm{E}-07$ \\
\hline Ap1 & Complex & Activated & 2.789 & $2.38 \mathrm{E}-07$ \\
\hline IL22 & Cytokine & Activated & 3.199 & 4.46E-07 \\
\hline Vegf & Group & Activated & 3.595 & 4.97E-07 \\
\hline MAP2K3 & Kinase & Activated & 2.776 & $5.32 \mathrm{E}-07$ \\
\hline NfkB-RelA & Complex & Activated & 2.107 & $5.68 \mathrm{E}-07$ \\
\hline ERK1/2 & Group & Activated & 4.027 & $1.03 \mathrm{E}-06$ \\
\hline EGFR & Kinase & Activated & 3.463 & 1.17E-06 \\
\hline IFI16 & Transcription regulator & Activated & 2.427 & $1.44 \mathrm{E}-06$ \\
\hline E2F2 & Transcription regulator & Activated & 2.634 & $1.82 \mathrm{E}-06$ \\
\hline ZFP36 & Transcription regulator & Inhibited & -3.606 & $2.12 \mathrm{E}-06$ \\
\hline
\end{tabular}

Predicted activity based on gene expression values in canine iUC relative to normal bladder. A z-score $>2$ indicates activation, while a $z$-score $<-2$ indicates inhibition

EP2 signaling promotes tumor development via multiple mechanisms, including stimulatory effects on cell proliferation, survival, invasion, angiogenesis, and metastasis [36]. A recent study demonstrated that $\mathrm{PGE}_{2}$ induces programmed cell death protein ligand 1 expression in tumor-associated macrophages and myeloidderived suppressor cells, leading to the inhibition of anti-tumor immunity [37]. Furthermore, $\mathrm{PGE}_{2}$ is involved in resistance to chemotherapy by promoting repopulation of cancer stem cells in bladder cancer [38]. In addition, COX-2 inhibitors consistently exert antitumor effects on bladder cancer in both dogs and humans [7, 24, 39]. These findings suggest that the $\mathrm{COX}-2 / \mathrm{PGE}_{2} / \mathrm{EP} 2$ pathway plays a role in tumorigenesis and progression of bladder cancer.
ERBB2 (also known as HER2) was the second most activated upstream regulator in canine iUC. HER2 is a membrane-bound receptor tyrosine kinase belonging to the epidermal growth factor receptor (EGFR) family and is implicated as an oncogene in many human cancers [40]. Notably, HER2 is amplified and overexpressed in one-third of patients with breast cancer and is associated with poor prognosis [41]. HER2 overexpression facilitates excessive dimer formation, driving oncogenic cell survival, proliferation, apoptosis suppression, and angiogenesis [40, 42]. Thus, a number of clinical trials of HER2-targeted therapies in patients with breast cancer have been conducted, showing increased survival in both the metastatic and early-stage settings of the disease [40, 43-45]. 
Table $\mathbf{3}$ Top biological functions of differentially expressed genes in canine iUC

\begin{tabular}{llll}
\hline Biological functions & Predicted activation state & Bias-corrected z-score & $P$-value \\
\hline Cancer & Activated & 2.051 & $2.08 \mathrm{E}-22$ \\
Epithelial cancer & Activated & 2.834 & $5.93 \mathrm{E}-21$ \\
Neoplasia of epithelial tissue & Activated & 2.221 & $7.60 \mathrm{E}-21$ \\
Tumorigenesis of tissue & Activated & 2.21 & $1.10 \mathrm{E}-20$ \\
Abdominal neoplasm & Activated & 2.057 & $2.58 \mathrm{E}-19$ \\
Proliferation of cells & Activated & 4.057 & $3.16 \mathrm{E}-14$ \\
Cell movement of leukocytes & Activated & 2.678 & $6.35 \mathrm{E}-10$ \\
Function of muscle & Inhibited & -2.234 & $9.06 \mathrm{E}-09$ \\
Mammary tumor & Activated & 2.228 & $3.46 \mathrm{E}-08$ \\
Contractility of muscle & Inhibited & -3.244 & $4.22 \mathrm{E}-08$ \\
\hline
\end{tabular}

Predicted activity based on gene expression values in canine iUC relative to healthy bladder. A z-score $>2$ indicates activation, while a $z$-score $<-2$ indicates inhibition

Recently, The Cancer Genome Atlas project reported mutation or amplification of ERBB2 in a subset of human iUC [26]. Another study also identified activating ERBB2 mutations in the absence of gene amplification in human iUC [46]. These observations

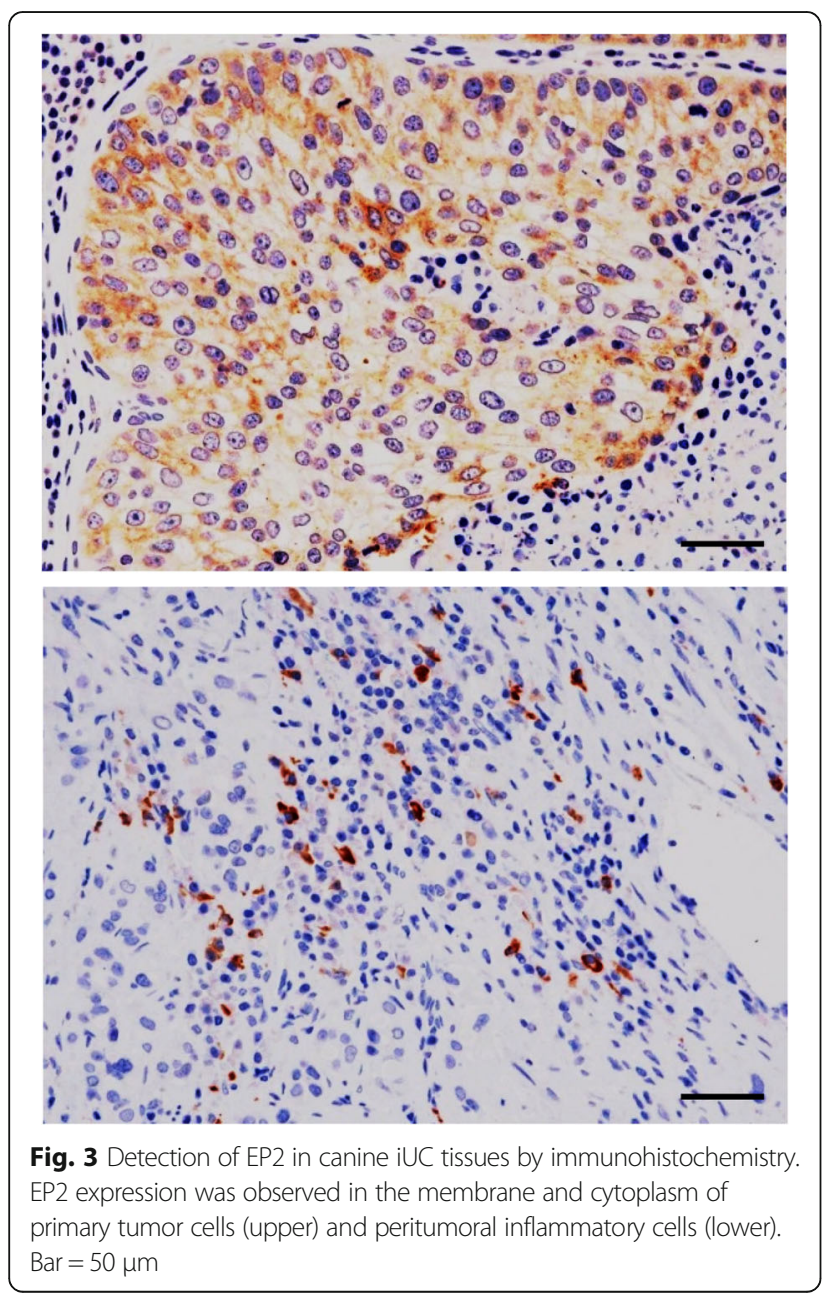

suggest that HER2-targeted therapy might also be effective for the treatment of iUC. Clinical trials to evaluate the efficacy and safety of HER2-targeted drugs in patients with bladder cancer are ongoing [47]. Our data indicate that HER2 is involved in disease progression and is a therapeutic target in canine iUC, as is the case with humans.

Pathways of several transcription factors (CCND1, FOXM1, E2F3, FOXO1, and JUN) were enriched in canine iUC. Of these, CCND1, which encodes cyclin D1, plays a critical role in the cell cycle machinery (e.g., G1-S transition) and is considered a promising target for cancer therapy [48]. Proinflammatory and angiogenesis cytokines (IL6, IL1, CSF2, TNF, IFNG, IL22, HGF, and Vegf) were also enriched in canine iUC. Our data support that aberrant inflammation and neovascularization occur in the tumor environment, and that anti-inflammatory and anti-angiogenic drugs targeting these cytokines have therapeutic potential for canine iUC [49]. Recently, immune-checkpoint inhibitors targeting the programmed death 1/programmed death-ligand 1 (PD-1/PD-L1), atezolizumab and nivolumab, have been approved by the Food and Drug Administration (FDA) in patients with advanced bladder cancer [50]. Although our study did not detect PD-L1 (CD274) within the 2513 DEGs, immune-checkpoint inhibitors might be effective against canine iUC.

By contrast, this study predicted that the pathways of Irgm1, TP53, let-7, and ZFP36 are inhibited in canine iUC. Irgm1 is a member of the interferon- $\gamma($ IFN- $\gamma$ )-inducible GTPase family and plays a role in innate immunity by regulating autophagy [51]. Recent studies have reported that Irgm1 enhances tumorigenesis and metastasis of melanoma in mice [52, 53]. High concentrations of IFN- $\gamma$ are toxic to neighbor cells; however, Irgm 1 protects immune cells that produce IFN- $\gamma$ from autophagic cell death, thereby promoting the expansion of $\mathrm{CD}^{+} \mathrm{T}$ cells [54]. Although the significance of Irgm1 
in canine iUC is unclear, inhibition of the pathway might prevent anti-tumor immunity from eliminating cancer cells through enhancement of IFN- $\gamma$-induced immune cell death.

TP53 is one of the most important tumor-suppressor genes and plays a role in cell cycle arrest and apoptosis in response to DNA damage [55]. In humans, about half of all tumor types including bladder cancer possess alterations in TP53 or its upstream/downstream genes [26, 56]. In dogs, TP53 mutations are also observed in many types of tumors, such as lymphoma [57], osteosarcoma $[58,59]$, and mammary tumor $[60,61]$. In canine iUC, the presence of TP53 mutation is deduced by immunohistochemistry (IHC). The mutated p53 protein, with a prolonged half-life, is considered to result in positive immunoreactivity of neoplastic cell nuclei, and thus can be detected by IHC [62]. However, TP53 mutations have not been directly confirmed in canine iUC by DNA sequencing analysis. Considering that the TP53 pathway was inhibited in this study, genetic and/or epigenetic abnormalities of TP53 may be present in canine iUC.

let-7 is a founding member of the microRNA (miRNA) family, postulated to function as a tumor suppressor gene by negatively regulating the post-transcriptional expression of multiple oncogenes including Ras, Myc, and Hmga2, as well as other cell cycle regulator genes in humans [63-65]. Reduced expression of let-7 is often observed and associated with poor prognosis in many human cancers, particularly in lung carcinoma [66]. Furthermore, let-7 has an attractive potential for the treatment of cancers; for example, intranasal let-7 administration reduced tumor formation in a mouse model of lung cancer [67]. As the let-7 pathway was significantly inhibited while the Ras pathway was significantly activated in canine iUC (Additional file 7: Table S4), exogenous let-7 administration may restore the reduced expression of let7 and may be a potent therapeutic strategy.

ZFP36 encodes a tandem Cys-Cys-Cys-His zinc finger protein (also known as tristetraprolin) that binds to and promotes degradation of AU-rich elements in the 3 '-untranslated regions of certain mRNAs [68]. COX-2 is a well-established target of ZFP36 [69, 70], and in this study, we found that dogs with iUC exhibited suppression of the ZFP36 pathway. Thus, overexpression of COX-2 in canine iUC $[30,31]$ could be attributed to the inhibition of the ZFP36 pathway and subsequent lack of rapid mRNA degradation.

Highly expressed genes present in canine iUC were associated with cancer and leukocyte migration, whereas downregulated genes were associated with muscle function. Decreased expression of muscle-related genes may result in a muscle-invasive phenotype. In addition, gene sets enriched in canine iUC were associated with mammary tumors, consistent with previous studies whereby signatures of human bladder cancer are similar to that of breast cancer [26, 71].

Several biomarkers can be used as a screening test for bladder cancer in dogs as well as humans [72]. Urinary bladder tumor antigen (BTA) test is an agglutination reaction that detects complexes of basement membrane proteins degraded by proteinases of urothelial neoplasms [73]. The present study revealed that the mRNA expressions of matrix metalloproteinase 7 (MMP7), MMP9, MMP12, and MMP13 were significantly upregulated in canine iUC (Additional file 3: Table S2). A recent study observed significantly high rates of the BRAF V595E mutation (approximately $80 \%$ ) in canine iUC and prostate cancer [13]. In our study, this mutation was detected in 6/11 (54.5\%) cases, although no clear separation was observed between dogs with or without BRAF mutation in a PCA plot. A possible reason may be that the sample size was too small to detect a significant correlation; therefore, more extensive studies are needed to clarify the role of BRAF mutation in canine iUC.

One limitation of this study is that the healthy control dogs were not well matched to the iUC cases by sex and breed, although there was no significant difference in the sex distribution between the two groups. In the pathway analysis of this study, sex hormone-associated genes were not observed in the top 500 upstream regulators of DEGs (Additional file 7: Table S4). Therefore, it appears that gender bias did not impact the RNA-Seq data. Since it was impossible to obtain fresh bladder tissues from various breeds matched to the clinical cases, all healthy control dogs were the same breed, and this could lead to erroneous results related to the expression of some genes that differ between breeds. Further studies will be necessary to examine breed differences in bladder gene expression.

\section{Conclusions}

In conclusion, alteration of multiple pathways in canine iUC was uncovered by RNA-Seq analysis. Upstream regulators of the altered pathways documented here are potential therapeutic and diagnostic targets for canine iUC. Our data confirmed the similarities in gene sets between dogs and humans, indicating the value of spontaneous canine iUC as a relevant animal model for human bladder cancer.

\section{Additional files}

Additional file 1: Table S1. Sequences of the oligonucleotide primers used for quantitative PCR. (XLSX $11 \mathrm{~kb})$

Additional file 2: Figure S1. Principal component analysis (PCA) plot of canine iUC with or without BRAF V595E mutation. The PCA plot did not show clear separation between cases with (red) or without (blue) the BRAF mutation. (TIF $4649 \mathrm{~kb}$ ) 
Additional file 3: Table S2. Top 500 upregulated differentially expressed genes (DEGs) in canine iUC. (XLSX 26 kb)

Additional file 4: Table S3. Top 500 downregulated differentially expressed genes (DEGs) in canine iUC. (XLSX 25 kb)

Additional file 5: Figure S2. Hierarchical clustering of the genes that were previously shown to divide dogs with iUC into subgroups. Analysis yielded three clusters composed of five healthy controls, those of two dogs with iUC (iUC1 and iUC11), and those of the remaining nine iUC cases. Genes indicated in blue are down-regulated, while genes indicated in red are upregulated. (TIF 7001 kb)

Additional file 6: Figure S3. mRNA expression of top 5 DEGs in the bladder of healthy dogs and dogs with iUC. TBP was used as an internal control. Open and closed circles represent healthy dogs and dogs with iUC, respectively. ${ }^{*} P<0.05$, ${ }^{* *} P<0.01$. (TIF $7016 \mathrm{~kb}$ )

Additional file 7: Table S4. Top 500 upstream regulators of differentially expressed genes in canine iUC. Predicted activity based on gene expression values in canine iUC relative to normal bladder. A z-score $>2$ indicates activation, while a z-score $<-2$ indicates inhibition. (XLSX $17 \mathrm{~kb}$ )

\section{Abbreviations}

COX: Cyclooxygenase; DEGs: Differentially expressed genes; EGFR: Epiderma growth factor receptor; IFN-y: Interferon- $\gamma$; iUC: Invasive urothelial carcinoma; miRNA: MicroRNA; PCA: Principal component analysis; $P E_{2}$ : Prostaglandin $E_{2}$; RNA-Seq: RNA sequencing

\section{Funding}

This work was funded by the Grant-in-Aid for Scientific Research from JSPS (KAKENHI Grant Numbers $16 \mathrm{H} 06208$ and 17H05043) and the Anicom Capital Research Grant (EVOLVE). The funding body had no role in the design of the study and collection, analysis, and interpretation of data and in writing the manuscript.

\section{Availability of data and materials}

The datasets used and/or analysed during the current study are available from the corresponding author on reasonable request and will also be available at the DDBJ Sequenced Read Archive repository (http:// trace.ddbj.nig.ac.jp/dra/index_e.html) with accession number DRA005844.

\section{Authors' contributions}

SM designed and performed the experiments, analyzed the data, and wrote the manuscript. HT analyzed the data and wrote the manuscript. Al, MT, Gl, and TU performed the experiments. TY and NM assisted in experimental conception and design and helped draft the manuscript. All authors read and approved the final manuscript.

\section{Ethics approval and consent to participate}

The study protocol of tumor tissue sampling was approved by the anima care committee of the Veterinary Medical Center of the University of Tokyo, and written informed consent was obtained from all dog owners. The use of experimental animals in this study was approved by the animal care committee of the University of Tokyo (approval no. P11-530).

\section{Competing interests}

The authors declare that they have no competing interests.

\section{Publisher's Note}

Springer Nature remains neutral with regard to jurisdictional claims in published maps and institutional affiliations.

\section{Author details}

'Department of Veterinary Clinical Pathobiology, Graduate School of Agricultural and Life Sciences, The University of Tokyo, Tokyo, Japan. Veterinary Medical Center, Graduate School of Agricultural and Life Sciences, The University of Tokyo, Tokyo, Japan. Department of Veterinary Pathology, Graduate School of Agricultural and Life Sciences, The University of Tokyo, Tokyo, Japan. ${ }^{4}$ Anicom Specialty Medical Institute Inc., Tokyo, Japan.
Received: 17 June 2017 Accepted: 18 April 2018

Published online: 27 April 2018

\section{References}

1. Torre LA, Bray F, Siegel RL, Ferlay J, Lortet-Tieulent J, Jemal A. Global cancer statistics, 2012. CA Cancer J Clin. 2015;65(2):87-108.

2. Kaufman DS, Shipley WU, Feldman AS. Bladder cancer. Lancet. 2009; 374(9685):239-49.

3. Loehrer PJ Sr, Einhorn LH, Elson PJ, Crawford ED, Kuebler P, Tannock I, Raghavan D, Stuart-Harris R, Sarosdy MF, Lowe BA, et al. A randomized comparison of cisplatin alone or in combination with methotrexate, vinblastine, and doxorubicin in patients with metastatic urothelial carcinoma: a cooperative group study. J Clin Oncol. 1992;10(7):1066-73.

4. Galsky MD, Hahn NM, Rosenberg J, Sonpavde G, Hutson T, Oh WK, Dreicer R, Vogelzang N, Sternberg CN, Bajorin DF, et al. Treatment of patients with metastatic urothelial cancer "unfit" for Cisplatin-based chemotherapy. J Clin Oncol. 2011;29(17):2432-8.

5. Kobayashi T, Owczarek TB, McKiernan JM, Abate-Shen C. Modelling bladder cancer in mice: opportunities and challenges. Nat Rev Cancer. 2015:15(1):42-54

6. Knapp DW, Glickman NW, Denicola DB, Bonney PL, Lin TL, Glickman LT. Naturally-occurring canine transitional cell carcinoma of the urinary bladder a relevant model of human invasive bladder cancer. Urol Oncol. 2000:5(2):47-59.

7. Knapp DW, Ramos-Vara JA, Moore GE, Dhawan D, Bonney PL, Young KE. Urinary bladder cancer in dogs, a naturally occurring model for cancer biology and drug development. ILAR J. 2014;55(1):100-18.

8. Dhawan D, Paoloni M, Shukradas S, Choudhury DR, Craig BA, Ramos-Vara JA, Hahn N, Bonney PL, Khanna C, Knapp DW. Comparative gene expression analyses identify luminal and basal subtypes of canine invasive Urothelial carcinoma that mimic patterns in human invasive bladder cancer. PLoS One. 2015;10(9):e0136688.

9. Mooney M, Bond J, Monks N, Eugster E, Cherba D, Berlinski P, Kamerling S, Marotti K, Simpson H, Rusk T, et al. Comparative RNA-Seq and microarray analysis of gene expression changes in B-cell lymphomas of Canis familiaris. PLoS One. 2013;8(4):e61088.

10. Sultan M, Schulz MH, Richard H, Magen A, Klingenhoff A, Scherf M, Seifert M, Borodina T, Soldatov A, Parkhomchuk D, et al. A global view of gene activity and alternative splicing by deep sequencing of the human transcriptome. Science. 2008;321(5891):956-60.

11. Meuten D, Everitt J, Inskeep W, RM, MP J, Thompson K. Urinary bladder tumors: histological classification of tumors of the urinary system of domestic animal. Washington, DC: Armed Forces Institute of Pathology and the World Health Organization; 2004.

12. Valli VE, Norris A, Jacobs RM, Laing E, Withrow S, Macy D, Tomlinson J, McCaw D, Ogilvie GK, Pidgeon G, et al. Pathology of canine bladder and urethral cancer and correlation with tumour progression and survival. J Comp Pathol. 1995:113(2):113-30.

13. Mochizuki H, Shapiro SG, Breen M. Detection of BRAF mutation in urine DNA as a molecular diagnostic for canine Urothelial and prostatic carcinoma. PLoS One. 2015;10(12):e0144170.

14. Bolger AM, Lohse M, Usadel B. Trimmomatic: a flexible trimmer for Illumina sequence data. Bioinformatics. 2014;30(15):2114-20.

15. Langmead B, Salzberg SL. Fast gapped-read alignment with bowtie 2 . Nat Methods. 2012;9(4):357-9.

16. Trapnell C, Williams BA, Pertea G, Mortazavi A, Kwan G, van Baren MJ, Salzberg SL, Wold BJ, Pachter L. Transcript assembly and quantification by RNA-Seq reveals unannotated transcripts and isoform switching during cell differentiation. Nat Biotechnol. 2010;28(5):511-5

17. Robinson MD, McCarthy DJ, Smyth GK. edgeR: a bioconductor package for differential expression analysis of digital gene expression data. Bioinformatics. 2010;26(1):139-40.

18. Zhou X, Lindsay H, Robinson MD. Robustly detecting differential expression in RNA sequencing data using observation weights. Nucleic Acids Res. 2014; 42(11):e91.

19. Robinson MD, Oshlack A. A scaling normalization method for differential expression analysis of RNA-seq data. Genome Biol. 2010;11(3):R25.

20. Maeda S, Ohno K, Nakamura K, Uchida K, Nakashima K, Fukushima K, Nakajima M, Goto-Koshino Y, Fujino Y, Tsujimoto H. Increased expression of fractalkine and its receptor $\mathrm{CX}(3) \mathrm{CR} 1$ in canine inflammatory bowel disease 
and their possible role in recruitment of intraepithelial lymphocytes. Vet Immunol Immunopathol. 2012;148(3-4):226-35.

21. Maeda S, Ohno K, Nakamura K, Uchida K, Nakashima K, Fukushima K, Tsukamoto A, Goto-Koshino Y, Fujino Y, Tsujimoto H. Mucosal imbalance of interleukin-1 beta and interleukin-1 receptor antagonist in canine inflammatory bowel disease. Vet J. 2012;194:66-70.

22. Millanta F, Asproni P, Canale A, Citi S, Poli A. COX-2, mPGES-1 and EP2 receptor immunohistochemical expression in canine and feline malignant mammary tumours. Vet Comp Oncol. 2016;14(3):270-80.

23. Millanta F, Asproni P, Cancedda S, Vignoli M, Bacci B, Poli A. Immunohistochemical expression of COX-2, mPGES and EP2 receptor in normal and reactive canine bone and in canine osteosarcoma. J Comp Pathol. 2012;147(2-3):153-60.

24. Fulkerson CM, Knapp DW. Management of transitional cell carcinoma of the urinary bladder in dogs: a review. Vet J. 2015;205(2):217-25.

25. Owen LN. TNM classification of Tumours in domestic animals. Geneva: World Health Organization (WHO); 1980

26. Cancer Genome Atlas Research N. Comprehensive molecular characterization of urothelial bladder carcinoma. Nature. 2014;507(7492): 315-22.

27. Shimizu T. Lipid mediators in health and disease: enzymes and receptors as therapeutic targets for the regulation of immunity and inflammation. Annu Rev Pharmacol Toxicol. 2009;49:123-50.

28. Sugimoto Y, Narumiya S. Prostaglandin E receptors. J Biol Chem. 2007; 282(16):11613-7.

29. Greenhough A, Smartt HJ, Moore AE, Roberts HR, Williams AC, Paraskeva C, Kaidi A. The COX-2/PGE2 pathway: key roles in the hallmarks of cancer and adaptation to the tumour microenvironment. Carcinogenesis. 2009;30(3):377-86.

30. Cekanova M, Uddin MJ, Bartges JW, Callens A, Legendre AM, Rathore K, Wright L, Carter A, Marnett LJ. Molecular imaging of cyclooxygenase-2 in canine transitional cell carcinomas in vitro and in vivo. Cancer Prev Res (Phila). 2013;6(5):466-76.

31. Khan KN, Knapp DW, Denicola DB, Harris RK. Expression of cyclooxygenase-2 in transitional cell carcinoma of the urinary bladder in dogs. Am J Vet Res. 2000;61(5):478-81.

32. Mohammed SI, Coffman K, Glickman NW, Hayek MG, Waters DJ, Schlittler D, DeNicola DB, Knapp DW. Prostaglandin E2 concentrations in naturally occurring canine cancer. Prostaglandins Leukot Essent Fatty Acids. 2001:64(1):1-4.

33. Mohammed SI, Knapp DW, Bostwick DG, Foster RS, Khan KN, Masferrer JL, Woerner BM, Snyder PW, Koki AT. Expression of cyclooxygenase-2 (COX-2) in human invasive transitional cell carcinoma (TCC) of the urinary bladder. Cancer Res. 1999:59(22):5647-50.

34. Shirahama T. Cyclooxygenase-2 expression is up-regulated in transitional cell carcinoma and its preneoplastic lesions in the human urinary bladder Clin Cancer Res. 2000;6(6):2424-30.

35. Wadhwa P, Goswami AK, Joshi K, Sharma SK. Cyclooxygenase-2 expression increases with the stage and grade in transitional cell carcinoma of the urinary bladder. Int Urol Nephrol. 2005;37(1):47-53.

36. Jiang J, Dingledine R. Prostaglandin receptor EP2 in the crosshairs of antiinflammation, anti-cancer, and neuroprotection. Trends Pharmacol Sci. 2013; 34(7):413-23.

37. Prima V, Kaliberova LN, Kaliberov S, Curiel DT, Kusmartsev S. COX2/ MPGES1/PGE2 pathway regulates PD-L1 expression in tumor-associated macrophages and myeloid-derived suppressor cells. Proc Natl Acad Sci U S A. 2017;114(5):1117-22

38. Kurtova AV, Xiao J, Mo Q, Pazhanisamy S, Krasnow R, Lerner SP, Chen F, Roh TT, Lay E, Ho PL, et al. Blocking PGE2-induced tumour repopulation abrogates bladder cancer chemoresistance. Nature. 2015;517(7533):209-13.

39. Dhawan D, Craig BA, Cheng L, Snyder PW, Mohammed SI, Stewart JC, Zheng R, Loman RA, Foster RS, Knapp DW. Effects of short-term celecoxib treatment in patients with invasive transitional cell carcinoma of the urinary bladder. Mol Cancer Ther. 2010;9(5):1371-7.

40. Baselga J, Swain SM. Novel anticancer targets: revisiting ERBB2 and discovering ERBB3. Nat Rev Cancer. 2009;9(7):463-75.

41. Ursini-Siegel J, Schade B, Cardiff RD, Muller WJ. Insights from transgenic mouse models of ERBB2-induced breast cancer. Nat Rev Cancer. 2007; 7(5):389-97.

42. Yarden $Y$, Sliwkowski MX. Untangling the ErbB signalling network. Nat Rev Mol Cell Biol. 2001;2(2):127-37.
43. Baselga J, Cortes J, Kim SB, Im SA, Hegg R, Im YH, Roman L, Pedrini JL, Pienkowski T, Knott A, et al. Pertuzumab plus trastuzumab plus docetaxel for metastatic breast cancer. N Engl J Med. 2012;366(2):109-19.

44. Kawajiri H, Takashima T, Kashiwagi S, Noda S, Onoda N, Hirakawa K. Pertuzumab in combination with trastuzumab and docetaxel for HER2-positive metastatic breast cancer. Expert Rev Anticancer Ther. 2015;15(1):17-26.

45. Moya-Horno I, Cortes J. The expanding role of pertuzumab in the treatment of HER2-positive breast cancer. Breast Cancer (Dove Med Press). 2015:7:125-32.

46. Ross JS, Wang K, Gay LM, Al-Rohil RN, Nazeer T, Sheehan CE, Jennings TA, Otto GA, Donahue A, He J, et al. A high frequency of activating extracellular domain ERBB2 (HER2) mutation in micropapillary urothelial carcinoma. Clin Cancer Res. 2014;20(1):68-75.

47. Carneiro BA, Meeks JJ, Kuzel TM, Scaranti M, Abdulkadir SA, Giles FJ. Emerging therapeutic targets in bladder cancer. Cancer Treat Rev. 2015;41(2):170-8.

48. Otto T, Sicinski P. Cell cycle proteins as promising targets in cancer therapy. Nat Rev Cancer. 2017;17(2):93-115.

49. Coussens LM, Werb Z. Inflammation and cancer. Nature. 2002;420(6917):860-7.

50. Farina MS, Lundgren KT, Bellmunt J. Immunotherapy in Urothelial cancer: recent results and future perspectives. Drugs. 2017;77(10):1077-89.

51. Howard J. The IRG proteins: a function in search of a mechanism. Immunobiology. 2008;213(3-4):367-75.

52. Dong H, Tian L, Li R, Pei C, Fu Y, Dong X, Xia F, Wang C, Li W, Guo X, et al. IFNg-induced Irgm1 promotes tumorigenesis of melanoma via dual regulation of apoptosis and Bif-1-dependent autophagy. Oncogene. 2015;34(42):5363-71.

53. Tian L, Li L, Xing W, Li R, Pei C, Dong X, Fu Y, Gu C, Guo X, Jia Y, et al. IRGM1 enhances B16 melanoma cell metastasis through PI3K-Rac1 mediated epithelial mesenchymal transition. Sci Rep. 2015;5:12357.

54. Feng CG, Zheng L, Jankovic D, Bafica A, Cannons JL, Watford WT, Chaussabel D, Hieny S, Caspar P, Schwartzberg PL, et al. The immunityrelated GTPase Irgm1 promotes the expansion of activated CD4+ T cell populations by preventing interferon-gamma-induced cell death. Nat Immunol. 2008;9(11):1279-87.

55. Blattner C. Regulation of p53: the next generation. Cell Cycle. 2008;7(20): 3149-53.

56. Vousden $\mathrm{KH}$, Lu X. Live or let die: the cell's response to p53. Nat Rev Cancer. 2002;2(8):594-604

57. Koshino A, Goto-Koshino Y, Setoguchi A, Ohno K, Tsujimoto H. Mutation of p53 gene and its correlation with the clinical outcome in dogs with lymphoma. J Vet Intern Med. 2016;30(1):223-9.

58. Johnson AS, Couto CG, Weghorst CM. Mutation of the p53 tumor suppressor gene in spontaneously occurring osteosarcomas of the dog. Carcinogenesis. 1998;19(1):213-7.

59. van Leeuwen IS, Cornelisse CJ, Misdorp W, Goedegebuure SA, Kirpensteijn J, Rutteman GR. P53 gene mutations in osteosarcomas in the dog. Cancer Lett. 1997;111(1-2):173-8.

60. Lee $\mathrm{CH}$, Kweon OK. Mutations of p53 tumor suppressor gene in spontaneous canine mammary tumors. J Vet Sci. 2002;3(4):321-5.

61. Wakui S, Muto T, Yokoo K, Yokoo R, Takahashi H, Masaoka T, Hano H, Furusato M. Prognostic status of p53 gene mutation in canine mammary carcinoma. Anticancer Res. 2001;21(1B):611-6.

62. Mutsaers AJ, Widmer WR, Knapp DW. Canine transitional cell carcinoma. J Vet Intern Med. 2003;17(2):136-44.

63. Chang TC, Yu D, Lee YS, Wentzel EA, Arking DE, West KM, Dang CV, Thomas-Tikhonenko A, Mendell JT. Widespread microRNA repression by Myc contributes to tumorigenesis. Nat Genet. 2008;40(1):43-50.

64. Johnson SM, Grosshans H, Shingara J, Byrom M, Jarvis R, Cheng A, Labourier E, Reinert KL, Brown D, Slack FJ. RAS is regulated by the let-7 microRNA family. Cell. 2005;120(5):635-47.

65. Mayr C, Hemann MT, Bartel DP. Disrupting the pairing between let-7 and Hmga2 enhances oncogenic transformation. Science. 2007;315(5818):1576-9.

66. Takamizawa J, Konishi H, Yanagisawa K, Tomida S, Osada H, Endoh $\mathrm{H}_{\text {, }}$ Harano T, Yatabe Y, Nagino M, Nimura Y, et al. Reduced expression of the let-7 microRNAs in human lung cancers in association with shortened postoperative survival. Cancer Res. 2004;64(11):3753-6.

67. Esquela-Kerscher A, Trang P, Wiggins JF, Patrawala L, Cheng A, Ford L, Weidhaas JB, Brown D, Bader AG, Slack FJ. The let-7 microRNA reduces tumor growth in mouse models of lung cancer. Cell Cycle. 2008;7(6):759-64.

68. Carballo E, Lai WS, Blackshear PJ. Feedback inhibition of macrophage tumor necrosis factor-alpha production by tristetraprolin. Science. 1998;281(5379): 1001-5. 
69. Sawaoka H, Dixon DA, Oates JA, Boutaud O. Tristetraprolin binds to the 3'untranslated region of cyclooxygenase-2 mRNA. A polyadenylation variant in a cancer cell line lacks the binding site. J Biol Chem. 2003;278(16): 13928-35.

70. Young LE, Sanduja S, Bemis-Standoli K, Pena EA, Price RL, Dixon DA. The mRNA binding proteins HuR and tristetraprolin regulate cyclooxygenase 2 expression during colon carcinogenesis. Gastroenterology. 2009;136(5):1669-79

71. Damrauer JS, Hoadley KA, Chism DD, Fan C, Tiganelli CJ, Wobker SE, Yeh JJ, Milowsky MI, Iyer G, Parker JS, et al. Intrinsic subtypes of high-grade bladder cancer reflect the hallmarks of breast cancer biology. Proc Natl Acad Sci U S A. 2014;111(8):3110-5.

72. Alvarez A, Lokeshwar VB. Bladder cancer biomarkers: current developments and future implementation. Curr Opin Urol. 2007;17(5):341-6.

73. Henry CJ, Tyler JW, McEntee MC, Stokol T, Rogers KS, Chun R, Garrett LD, McCaw DL, Higginbotham ML, Flessland KA, et al. Evaluation of a bladder tumor antigen test as a screening test for transitional cell carcinoma of the lower urinary tract in dogs. Am J Vet Res. 2003;64(8):1017-20.

Ready to submit your research? Choose BMC and benefit from:

- fast, convenient online submission

- thorough peer review by experienced researchers in your field

- rapid publication on acceptance

- support for research data, including large and complex data types

- gold Open Access which fosters wider collaboration and increased citations

- maximum visibility for your research: over $100 \mathrm{M}$ website views per year

At BMC, research is always in progress.

Learn more biomedcentral.com/submissions 